\title{
BMJ Open Adverse outcomes of (post-)term births and differences in professional organisational contexts: an integral descriptive system approach
}

\author{
Loes Harmsen, ${ }^{1}$ Hans Schaaf ${ }^{2}$
}

To cite: Harmsen L, Schaaf JH. Adverse outcomes of (post-)term births and differences in professional organisational contexts: an integral descriptive system approach. BMJ Open 2014;4: e006083. doi:10.1136/ bmjopen-2014-006083

- Prepublication history for this paper is available online. To view these files please visit the journal online (http://dx.doi.org/10.1136/ bmjopen-2014-006083).

Received 10 July 2014 Revised 21 October 2014 Accepted 28 October 2014

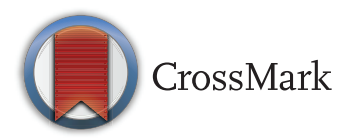

${ }^{1}$ Department of Obstetrics and Gynaecology, Deventer Hospital, Deventer,

The Netherlands

${ }^{2}$ Independent Researcher and Management Consultant, JHS Healthcare, Tilburg,

The Netherlands

Correspondence to Dr Loes Harmsen; harmsenl@dz.nl

\section{ABSTRACT}

Objective: The development and application of an integral descriptive model for monitoring and evaluation of patient flows and adverse outcomes of childbirth related to distinct categories of professional organisational contexts.

Setting: After categorisation of the individual professional contexts in which deliveries take place, the resulting framework has been superimposed on the 2002-2010 database of the Netherlands Perinatal Registry.

Participants: All Dutch hospitals and almost all first-line midwife practices recorded 1469955 (post-)term births from which only the patients with a spontaneous onset of labour ( $\mathrm{n}=1120508$ ) were included in a study on the quality of obstetric care outside office hours.

Main outcome measures: For the performance of professional organisational contexts the difference in relative incidence of perinatal death or Apgar score $<7$ between the related patient groups and the reference patient groups has been used. These differences have been expressed as risk ratio (RR) with a $95 \% \mathrm{Cl}$.

Results: Only the group of patients who started labour spontaneously under the supervision of the first-line midwife shows a proportional distribution over the parts of the day. In all other groups the distribution of patients is disproportional. The perinatal mortality rate declines by about $30 \%$ in the successive periods. This decline concerns mainly those patients who gave birth outside office hours.

Conclusions: The complexity and the dynamics of the obstetric care system make it virtually impossible to demonstrate fixed patterns in the relationships between the separate contextual factors and the (adverse) outcomes of births. To generate useful knowledge, it is necessary to evaluate changes in the obstetric care system periodically and systematically. Thus, the longitudinal application of the model demonstrated that the differences in perinatal mortality rate between the parts of the day have disappeared in recent years.

\section{INTRODUCTION}

Since its foundation in 1999, Euro-Peristat's objective has been to monitor and evaluate

\section{Strengths and limitations of this study}

We have developed a descriptive model helpful in the systematic monitoring and evaluation of the obstetric care system.

- Even if the attention is focused on a part of the obstetric care system, the entire system remains in view. The results of comparisons cannot be influenced by shifts between or exclusions of patient groups without being perceived.

- The design of the model is based on the most relevant organisational characteristics of the obstetric care system. In view of the system's dynamics we have opted for a combination of a transversal and a longitudinal study approach, while deliberately limiting the number of calendar years per distinct time period.

- The figures compared at the macro level consist of the sum of the figures that are collected at the meso level. Small differences in the relative incidence of adverse outcomes at the macro level may hide much larger differences at the meso level.

- The absolute numbers of adverse outcomes on which the differences in relative incidence are based are usually very small. In 2008-2010, the 'night/day difference' in the relative incidence of perinatal mortality in the total group of nonteaching hospitals corresponds to three cases on an annual basis.

maternal and child health during the perinatal period using valid and reliable indicators. Owing to the successive Peristat reports, ${ }^{1-3}$ the perinatal mortality rate has become a widely used indicator to compare the performance of obstetric care systems in the participating European countries. Partly as a result of the greatly increased attention to patient safety since the publication of the influential American report 'To Err is Human' ${ }^{4}$ the perinatal mortality rate is also often used to rule out or reveal differences in the safety of care within an obstetric care system. Thus, over the years, publications from different countries have reported a higher perinatal mortality rate at childbirth 
outside office hours than at delivery during the day. ${ }^{5-10}$ What almost all of these comparative studies have in common is that they take only part of the obstetric care system into consideration, have a transversal design, and are based on the data of a rather large number of mostly older calendar years. This poses the question: Is the design of these studies sufficiently consistent with the complexity and the dynamics that characterise each (obstetrical) care system?

\section{Professional organisational context}

The key concept in this study is the professional organisational context, defined here as the whole of knowledge, skills, organisational arrangements and technical facilities available to optimise the effectiveness and safety of (obstetric) care. The starting point is that the context of pregnancy and childbirth is determined by many interrelated factors. Each of these factors can exhibit incidental or structural deficiencies (whether or not through insufficient use) that contribute to substandard care and adverse outcomes. ${ }^{11}$ In our approach it is nevertheless essential that we consider the professional organisational context as a whole. This can be done at three levels. At the micro level, it concerns the context of an individual obstetric care process. At this level there are effectively as many professional organisational contexts as there are births. At the meso level, it concerns the context in a specific obstetric unit or ward. The focus of this study is on the macro level, the modelbased country-wide context that can be constructed using individual data. It is in the nature of each professional organisational context that it is far from stable. New insights, new regulations, new technologies, different allocations of tasks, revised professional standards, different forms of cooperation, new procedures and a wealth of other influences lead constantly to changes that affect the quality of care.

\section{Organisation of the obstetric care system}

In the Netherlands, the obstetric care system is based on the premise that pregnancy and childbirth are physiological phenomena. As long as there is no actual risk (ie, no manifest medical or obstetric problem) and the anamnesis (obstetric history, etc) is not seen as a potential risk, pregnancy and childbirth usually are supervised by a midwife (first line). Childbirth can take place either at a patient's home or in a maternity unit (mostly an annexe to a hospital). Once, however, the risk for mother and/or child is assessed as raised, supervision is transferred to an obstetrician in a general hospital (second line) or a (university) hospital with a neonatal intensive care unit (NICU) (third line). The organisational structure of the obstetric care system provides a functional stratification of professional organisational contexts (first, second, third line). Risk assessment and risk selection are the basis of virtually any contact between patient and care professional. The aim is primarily to find a fitting professional organisational context for each individual patient. Each contact can lead to an adjustment in context. The higher the assessed risk, the more requirements are imposed on the context in which pregnancy and childbirth are supervised. This means that the choice of the professional organisational context in which childbirth takes place is at least partly determined by the risk selection built into the obstetric care system.

\section{Categorisation of individual contexts}

Although obstetrics is practiced at the meso level, nearly all research into the contexts in which deliveries take place is geared towards fictitious contexts that are constructed at the macro level. ${ }^{5-10}$ In our approach the individual professional organisational contexts are categorised in such a way that they reflect the organisational structure of the national obstetric care system. Useful features for this are: the supervision of labour (first-line midwife and/or second or third line obstetrician), the location of birth and the part of the day in which the second stage of labour begins. ${ }^{12}$ To visualise the trends over time, the time period in which birth takes place is a useful starting point. While the individual contexts are categorised, the related patients (records) are simultaneously grouped at the macro level. The thus composed context related patient groups (subpopulations) are the core objects of our study. It is essential that the distinct context-categories and related patient groups are exhaustive and mutually exclusive. Each patient (record) is exclusively related to a single context category. This makes it possible, if required, to merge two or more context related patient groups and to consider these as a whole (figure 1).

\section{Risk profile of context related patient groups}

The way in which obstetric care is organised dictates that the professional organisational context of a delivery is largely determined by risk assessment and risk selection at the individual level. This means that the distribution of patients over the various contexts is at least partly selective in character. This is reflected in the actual risk profiles of the distinct context related patient groups (figure 2). It is plausible that a high percentage of patients at higher actual risk (in the absence of professional intervention) correlates with a high relative incidence of adverse outcomes. This implies that the same actual risks that influence the choice of the professional organisational context also influence the incidence of adverse outcomes in the related patient group. Hence, a professional organisational context-category cannot be considered as an independent determinant. It is important to note that the risk profile of a context related patient group can also be affected by the exclusion of patients (records) because of the design of a study.

\section{Distribution of deliveries over the $\mathbf{2 4} \mathrm{h}$ day}

The distribution of patients (records) over the distinct context-categories is not only determined by professional choices. This is especially true for patients with a 


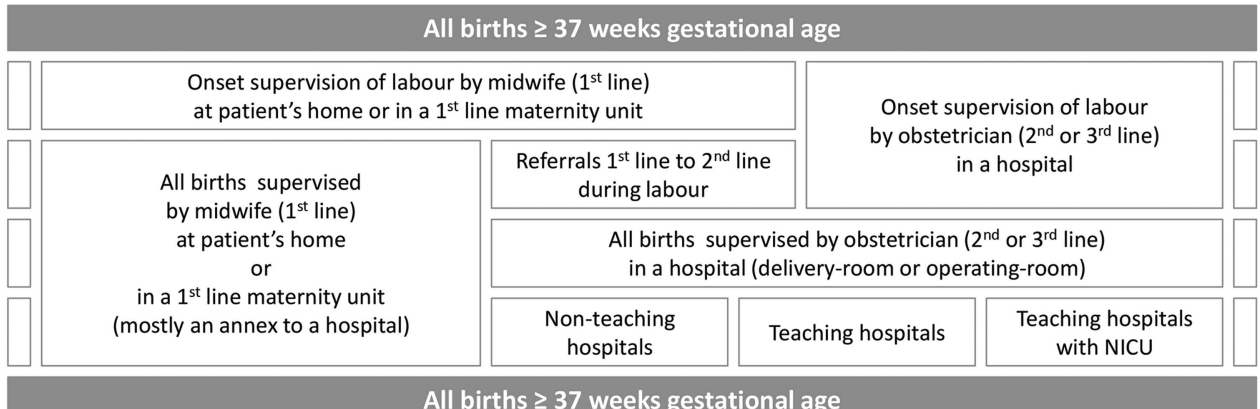

All births $\geq 37$ weeks gestational age

Figure 1 Overview of the main (merged) context-categories and related patient groups.

spontaneous onset of labour (ie, without professional intervention). We base this claim on a twofold assumption: (1) Under natural conditions the deliveries are randomly spread and, therefore, in a population of sufficient size, (approximately) equally distributed over the $24 \mathrm{~h}$ day; (2) Under these conditions, the actual and potential risks in this population are also (approximately) equally distributed over the $24 \mathrm{~h}$ day. The reverse side of this basic assumption is that, in case of an unequal distribution of deliveries, we cannot assume that the risks are equally distributed over those $24 \mathrm{~h}^{12}$ In this way we therefore ignore the smaller peaks and troughs in the distribution of births over the day that have been described. ${ }^{13} 14$

\section{A responsible comparison of context-categories}

If the objective is to gain insight into the performance of a professional organisational context-category, the conventional method is to compare the (adverse) outcomes of births in the related patient group (transversal) with those in a reference category. ${ }^{5-10}$ To complicate matters, the relative incidence of adverse outcomes in a context related patient group is not only affected by contextual factors, but also by patient-related factors (figure 2).
A difference in the incidence of adverse outcomes between two context related patient groups can therefore only be attributed (exclusively) to a difference in performance of the relevant professional organisational contexts if it can be established that this difference in incidence is not caused by a difference in actual risk profiles.

\section{Study objective}

The complexity and the dynamic character of the obstetric care system have inspired us to develop an integral descriptive model for the monitoring and evaluation of patient flows and of the adverse outcomes of (post-) term childbirth related to the distinct categories of professional organisational contexts. In this article, we have focused our attention on the obstetric care in hospitals and, more specifically, on the quality and safety of care outside office hours.

\section{MATERIALS AND METHODS}

The nationwide data for this study has been provided by the Netherlands Perinatal Registry (PRN). This PRN data collection is obtained through a validated coupling

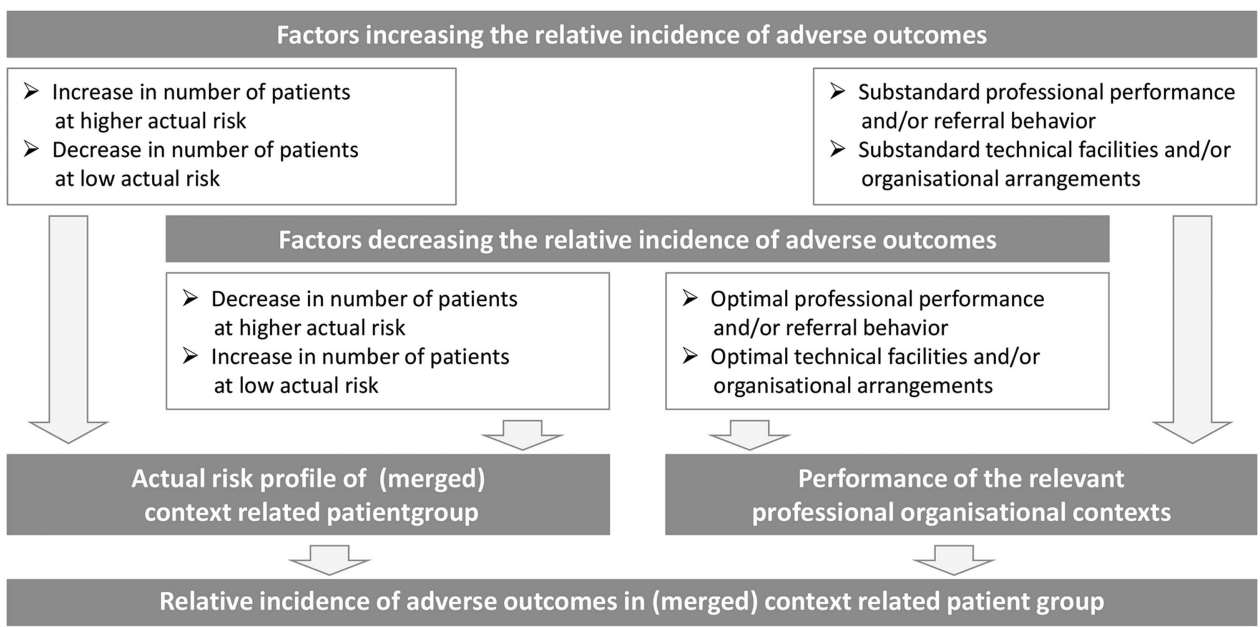

Figure 2 Factors influencing the relative incidence of adverse outcomes in context related patient groups. 
of three different registries: the midwifery registry (LVR1), the obstetrics registry (LVR2) and the neonatology registry (LNR). ${ }^{15}$ The PRN registry covers approximately $95 \%$ of all births in the Netherlands.

\section{Model of the obstetric care system}

The descriptive model of the obstetric care system we have developed as part of our study is based on the categorisation of individual professional organisational contexts and related patients (records). In the most detailed view of the model, the subsystems (and related subpopulations) correspond to the distinct context-categories and related patient groups. ${ }^{12}$ The more global model presented in this article has been obtained by merging a number of context-categories and related patient groups (figure 1). Determiners of the main (merged) contextcategories and related patient groups are the supervision of labour and the location of birth. In this global representation of the model we distinguish non-teaching hospitals, teaching hospitals (obstetrics and gynaecology) and teaching hospitals with a NICU. On the basis of the current timetables in healthcare, we made a distinction between the individual professional organisational contexts in the daytime (9:00 to 16:00) and the contexts during the evening and night (19:00 to 6:00). To establish as distinct a contrast as possible between the subgroups related to both these context categories, we have defined a third part of the day (category) for the contexts during the intermediate duty handovers in the early morning and the late afternoon. To mark the time of childbirth, we have used the onset of the second stage, the phase of labour immediately prior to birth. In this phase high demands are placed on the professional organisational context.

\section{Transversal and longitudinal comparisons}

In our study approach we do not restrict ourselves to a transversal comparison of the incidence of adverse outcomes in different context related patient groups, but combine this approach with the visualisation of developments in successive time periods. ${ }^{16}$ Considering that professional organisational contexts are constantly subject to change, we have chosen time periods of a limited number of calendar years. Using relatively recent PRN data, the longitudinal approach in this article covers three successive time periods: 2002-2004 (I), 2005-2007 (II) and 2008-2010 (III).

\section{Study population}

Our basic population includes all births with a gestational age $\geq 37$ weeks of the calendar years 2002-2010 $(n=1469$ 955). Excluded from the final study population are all cases of antepartum mortality $(n=2008)$. Also excluded are all elective caesarean sections, multiple pregnancies and/or inductions of labour $(n=347808)$. The elective caesarean sections and the inductions of labour are excluded because these obstetric interventions are not equally distributed over the $24 \mathrm{~h}$ day. We have called the resulting study population a Spontaneous onset of labour, after reaching the Term period, Alive at the onset of labour, Single child (STAS) population: patients who came into the STAS ( $n=1120$ $508)$. This STAS population corresponds with about $70 \%$ of the complete PRN data file.

\section{Testing the basic assumption}

Within the global model outlined above (figure 1) we distinguish a merged, context related patient group that is ideally suited to test the basic assumption: 'Onset supervision of labour by midwife (1st line)'. Characteristic of this group is that it includes all patients who were assessed as low risk during pregnancy and who came into a spontaneous onset of labour. Assuming an equal distribution of patients (records) over the $24 \mathrm{~h}$ day, the expected distribution over the distinct parts of the day is as follows: daytime $29.2 \%$ (7/24), evening/ night $45.8 \%(11 / 24)$ and duty handovers $25 \%$ (6/24).

\section{Outcomes of births}

The two outcome variables used in this study both have the character of adverse outcomes: the perinatal mortality rate and the incidence of the Apgar score $<7$ after 5 min. Partly due to the exclusion of antepartum mortality cases in the STAS population, the average incidence of perinatal mortality is especially very low-a reason for us to focus on the absolute numbers as well as the proportions (with a 95\% CI) of these variables. To determine the difference in incidence of adverse outcomes between two (merged) context related patient groups, we used the risk ratio (RR) with a $95 \%$ CI.

There are different reasons to question case-mix adjustment in a non-randomised observational evaluation study such as this. ${ }^{17}$ For example, the PRN registration does not provide for a clear and complete data set with respect to the actual risks during labour. The main reason why we have desisted from case-mix adjustment, however, is that it is not compatible with the descriptive deterministic nature of our study design.

\section{RESULTS}

For the presentation of its concrete applications we use tables that are directly derived from the described model (tables 1-3).

To enable targeted references to these tables, the columns are indicated by a capital letter and the rows with a number. Each of the uniform tables in this article covers one of the distinct time periods. The upper rows (1-3) of these tables show to what extent the excluded patient categories affect the size of the study population and the incidence of both outcome variables. The other rows (4-36) of the tables show how the number of patients (records) and the adverse outcomes within the STAS population are distributed among the main (merged) context related patient groups (figure 1). 
Table 1 Distribution of (post)-term births and associated adverse outcomes over the distinct (merged) context related patient (sub)groups in time period 2002-2004

\begin{tabular}{|c|c|c|c|c|c|c|c|c|c|c|c|c|c|c|}
\hline \multirow{2}{*}{\multicolumn{2}{|c|}{$\begin{array}{l}\text { Time period 2002-2004 (I) } \\
\text { Source: Netherlands Perinatal Registry }\end{array}$}} & \multicolumn{3}{|c|}{ Births (records) } & \multicolumn{3}{|c|}{ Incidence perinatal mortality ${ }^{4}$ ) } & \multicolumn{2}{|c|}{ Comparison \%o PMR (RR 95\% CI) ${ }^{5}$ ) } & \multicolumn{3}{|c|}{ Incidence Apgar score < 7 (5 min) } & \multicolumn{2}{|c|}{ Comparison \% Apgar < 7 (RR 95\% Cl) } \\
\hline & & Number & $\%$ & $\%$ & Number & $\%$ & $\% \quad(95 \% \mathrm{Cl})$ & Parts of the day & Time periods & Number & $\%$ & $\% \quad(95 \% \mathrm{Cl})$ & Parts of the day & Time periods \\
\hline 1 & All births $\geq 37$ weeks gestational age & 506002 & 100 & & 1616 & 100 & $3.19(3.04-3.35)$ & & Reference & 5558 & 100 & $11.0(10.7-11.3)$ & & Reference \\
\hline 2 & Excluded antepartum mortality & 846 & 0.2 & & 846 & 52.4 & & & & 846 & 15.2 & & & \\
\hline 3 & Other excluded patient categories ${ }^{1}$ ) & 117248 & 23.2 & & 279 & 17.3 & $2.38(2.12-2.68)$ & & Reference & 1495 & 26.9 & $12.8(12.1-13.4)$ & & Reference \\
\hline 4 & All STAS births ${ }^{2}$ ) & 387908 & 76.7 & & 491 & 30.4 & $1.27(1.15-1.38)$ & & Reference & 3217 & 57.9 & $8.3(8.0-8.6)$ & & Reference \\
\hline 5 & STAS births supervised by $1^{\text {st }}$ line & 184355 & 36.4 & 100 & 75 & 4.6 & $0.41(0.31-0.50)$ & & Reference & 711 & 12.8 & $3.9(3.6-4.1)$ & & Reference \\
\hline 6 & Daytime & 52087 & 10.3 & 28.3 & 14 & 0.9 & $0.27(0.13-0.41)$ & Reference & Reference & 177 & 3.2 & $3.4(2.9-3.9)$ & Reference & Reference \\
\hline 7 & Evening/night & 86571 & 17.1 & 47.0 & 45 & 2.8 & $0.52(0.37-0.67)$ & $1.93(1.06-3.52)$ & Reference & 380 & 6.8 & $4.4(4.0-4.8)$ & $1.29(1.08-1.54)$ & Reference \\
\hline 8 & Duty handover & 45697 & 9.0 & 24.8 & 16 & 1.0 & $0.35(0.18-0.52)$ & $1.30(0.64-2.67)$ & Reference & 154 & 2.8 & $3.4(2.8-3.9)$ & $0.99(0.80-1.23)$ & Reference \\
\hline 9 & Referrals $1^{{ }^{t} \mathrm{l}}$ line to $2^{\text {nd }}$ line during labour & 90897 & 18.0 & 100 & 200 & 12.4 & $2.20(1.90-2.50)$ & & Reference & 1226 & 22.1 & $13.5(12.7-14.2)$ & & Reference \\
\hline 10 & Daytime & 30661 & 6.1 & 33.7 & 64 & 4.0 & $2.09(1.58-2.60)$ & Reference & Reference & 346 & 6.2 & $11.3(10.1-12.5)$ & Reference & Reference \\
\hline 11 & Evening/night & 37527 & 7.4 & 41.3 & 81 & 5.0 & $2.16(1.69-2.63)$ & $1.03(0.75-1.43)$ & Reference & 566 & 10.2 & $15.1(13.9-16.3)$ & $1.34(1.17-1.53)$ & Reference \\
\hline 12 & Duty handover & 22709 & 4.5 & 25.0 & 55 & 3.4 & $2.42(1.78-3.06)$ & $1.16(0.81-1.66)$ & Reference & 314 & 5.6 & $13.8(12.3-15.4)$ & $1.23(1.05-1.43)$ & Reference \\
\hline 13 & Onset supervision of labour $1^{\text {st line }}$ & 275252 & 54.4 & 100 & 275 & 17.0 & $1.00(0.88-1.12)$ & & Reference & 1937 & 34.9 & $7.0(6.7-7.4)$ & & Reference \\
\hline 14 & Daytime & 82748 & 16.4 & 30.1 & 78 & 4.8 & $0.94(0.73-1.15)$ & Reference & Reference & 523 & 9.4 & $6.3(5.8-6.9)$ & Reference & Reference \\
\hline 15 & Evening/night & 124098 & 24.5 & 45.1 & 126 & 7.8 & $1.02(0.84-1.19)$ & $1.08(0.81-1.43)$ & Reference & 946 & 17.0 & $7.6(7.1-8.1)$ & $1.21(1.08-1.34)$ & Reference \\
\hline 16 & Duty handover & 68406 & 13.5 & 24.9 & 71 & 4.4 & $1.04(0.80-1.28)$ & $1.10(0.80-1.52)$ & Reference & 468 & 8.4 & $6.8(6.2-7.5)$ & $1.08(0.96-1.23)$ & Reference \\
\hline 17 & Onset supervision of labour $2^{\text {nd }} / 3^{\text {rd }}$ line & 112656 & 22.3 & 100 & 216 & 13.4 & $1.92(1.66-2.17)$ & & Reference & 1280 & 23.0 & $11.4(10.7-12.0)$ & & Reference \\
\hline 18 & Daytime & 38400 & 7.6 & 34.1 & 62 & 3.8 & $1.61(1.21-2.02)$ & Reference & Reference & 396 & 7.1 & $10.3(9.3-11.3)$ & Reference & Reference \\
\hline 19 & Evening/night & 46190 & 9.1 & 41.0 & 90 & 5.6 & $1.95(1.55-2.35)$ & $1.21(0.87-1.67)$ & Reference & 550 & 9.9 & $11.9(10.9-12.9)$ & $1.15(1.02-1.31)$ & Reference \\
\hline 20 & Duty handover & 28066 & 5.5 & 24.9 & 64 & 4.0 & $2.28(1.72-2.84)$ & $1.41(1.00-2.00)$ & Reference & 334 & 6.0 & $11.9(10.6-13.2)$ & $1.15(1.00-1.33)$ & Reference \\
\hline 21 & STAS births supervised by $2^{\text {nd }} / 3^{\text {rd }}$ line & 203553 & 40.2 & 100 & 416 & 25.7 & $2.04(1.85-2.24)$ & & Reference & 2506 & 45.1 & $12.3(11.8-12.8)$ & & Reference \\
\hline 22 & Daytime & 69061 & 13.6 & 33.9 & 126 & 7.8 & $1.82(1.51-2.14)$ & Reference & Reference & 742 & 13.4 & $10.7(10.0-11.5)$ & Reference & Reference \\
\hline 23 & Evening/night & 83717 & 16.5 & 41.1 & 171 & 10.6 & $2.04(1.74-2.35)$ & $1.12(0.89-1.41)$ & Reference & 1116 & 20.1 & $13.3(12.6-14.1)$ & $1.24(1.13-1.36)$ & Reference \\
\hline 24 & Duty handover & 50775 & 10.0 & 24.9 & 119 & 7.4 & $2.34(1.92-2.76)$ & $1.28(1.00-1.65)$ & Reference & 648 & 11.7 & $12.8(11.8-13.7)$ & $1.19(1.07-1.32)$ & Reference \\
\hline 25 & Non-teaching hospitals & 85782 & 17.0 & 100 & 166 & 10.3 & $1.94(1.64-2.23)$ & & Reference & 944 & 17.0 & $11.0(10.3-11.7)$ & & Reference \\
\hline 26 & Daytime & 30555 & 6.0 & 35.6 & 49 & 3.0 & $1.60(1.15-2.05)$ & Reference & Reference & 266 & 4.8 & $8.7(7.7-9.8)$ & Reference & Reference \\
\hline 27 & Evening/night & 33843 & 6.7 & 39.5 & 68 & 4.2 & $2.01(1.53-2.49)$ & $1.25(0.87-1.81)$ & Reference & 416 & 7.5 & $12.3(11.1-13.5)$ & $1.41(1.21-1.65)$ & Reference \\
\hline 28 & Duty handover & 21384 & 4.2 & 24.9 & 49 & 3.0 & $2.29(1.65-2.93)$ & $1.43(0.96-2.12)$ & Reference & 262 & 4.7 & $12.3(10.8-13.7)$ & $1.41(1.19-1.67)$ & Reference \\
\hline 29 & Teaching hospitals & 92873 & 18.4 & 100 & 173 & 10.7 & $1.86(1.59-2.14)$ & & Reference & 1182 & 21.3 & $12.7(12.0-13.5)$ & & Reference \\
\hline 30 & Daytime & 30674 & 6.1 & 33.0 & 55 & 3.4 & $1.79(1.32-2.27)$ & Reference & Reference & 358 & 6.4 & $11.7(10.5-12.9)$ & Reference & Reference \\
\hline 31 & Evening/night & 39099 & 7.7 & 42.1 & 68 & 4.2 & $1.74(1.33-2.15)$ & $0.97(0.68-1.38)$ & Reference & 524 & 9.4 & $13.4(12.3-14.5)$ & $1.14(1.00-1.31)$ & Reference \\
\hline 32 & Duty handover & 23100 & 4.6 & 24.9 & 50 & 3.1 & $2.16(1.57-2.76)$ & $1.21(0.82-1.77)$ & Reference & 300 & 5.4 & $13.0(11.5-14.5)$ & $1.11(0.96-1.30)$ & Reference \\
\hline 33 & Teaching hospitals with $\mathrm{NICU}^{3}$ ) & 24898 & 4.9 & 100 & 77 & 4.8 & $3.09(2.40-3.78)$ & & Reference & 380 & 6.8 & $15.3(13.7-16.8)$ & & Reference \\
\hline 34 & Daytime & 7832 & 1.5 & 31.5 & 22 & 1.4 & $2.81(1.64-3.98)$ & Reference & Reference & 118 & 2.1 & $15.1(12.4-17.8)$ & Reference & Reference \\
\hline 35 & Evening/night & 10775 & 2.1 & 43.3 & 35 & 2.2 & $3.25(2.17-4.32)$ & $1.16(0.68-1.97)$ & Reference & 176 & 3.2 & $16.3(13.9-18.7)$ & $1.08(0.86-1.37)$ & Reference \\
\hline 36 & Duty handover & 6291 & 1.2 & 25.3 & 20 & 1.2 & $3.18(1.79-4.57)$ & $1.13(0.62-2.07)$ & Reference & 86 & 1.5 & $13.7(10.8-16.5)$ & $0.91(0.69-1.20)$ & Reference \\
\hline & A & B & C & D & E & $\mathbf{F}$ & G & $\mathrm{H}$ & $\mathrm{K}$ & M & $\mathbf{N}$ & v & w & $\mathrm{z}$ \\
\hline & $\begin{array}{l}\left.{ }^{1}\right) \text { Other excluded patient categories: electi } \\
\text { multiple pregnancy and induction of labour } \\
{ }^{2} \text { ) STAS: Spontaneous onset of labour, after } \\
\text { period, Alive at the onset of labour, Single }\end{array}$ & $\begin{array}{l}\text { e caesarea } \\
\text { reaching tt } \\
\text { hild }\end{array}$ & $\begin{array}{l}\text { an secti } \\
\text { ne Tern }\end{array}$ & & $\begin{array}{l}\left.{ }^{3}\right) \text { NICU: } \\
\left.{ }^{4}\right) \text { Perinat } \\
\text { postparti } \\
\left.{ }^{5}\right) \text { PMR: P }\end{array}$ & $\begin{array}{l}\text { Neonat } \\
\text { tal mor } \\
\text { um mo } \\
\text { Perinat }\end{array}$ & $\begin{array}{l}\text { Intensive Care Unit } \\
\text { lity: antepartum m } \\
\text { ality up to and inclu } \\
\text { Mortality Rate }\end{array}$ & $\begin{array}{l}\text { rtality, intrapartur } \\
\text { ding } 7 \text { days after b }\end{array}$ & ortality and & $\begin{array}{c}\text { Onset se } \\
- \\
- \\
-\end{array}$ & $\begin{array}{l}\text { Cond st } \\
\text { Dayti } \\
\text { Even } \\
\text { Duty }\end{array}$ & $\begin{array}{l}\text { ge of labour / caesa } \\
\text { he: } 9.00 \text { to } 16.00 \\
\text { g/night: } 19.00 \text { to } 6 \\
\text { andover: } 6.00 \text { to } 9\end{array}$ & $\begin{array}{l}\text { arean section during } \\
.00 \\
.00 \text { and } 16.00 \text { to } 19.0\end{array}$ & \\
\hline
\end{tabular}


Table 2 Distribution of (post)-term births and associated adverse outcomes over the distinct (merged) context related patient (sub)groups in time period 2005-2007

\begin{tabular}{|c|c|c|c|c|c|c|c|c|c|c|c|c|c|c|}
\hline \multirow{2}{*}{\multicolumn{2}{|c|}{$\begin{array}{l}\text { Time period 2005-2007 (II) } \\
\text { Source: Netherlands Perinatal Registry }\end{array}$}} & \multicolumn{3}{|c|}{ Births (records) } & \multicolumn{3}{|c|}{ Incidence perinatal mortality ${ }^{4}$ ) } & \multicolumn{2}{|c|}{ Comparison \%o PMR (RR 95\% Cl) ${ }^{5}$ ) } & \multicolumn{3}{|c|}{ Incidence Apgar score < 7 (5 min) } & \multicolumn{2}{|c|}{ Comparison \% Apgar < 7 (RR 95\% Cl) } \\
\hline & & Number & $\%$ & $\%$ & Number & $\%$ & $\% \quad(95 \% \mathrm{Cl})$ & Parts of the day & Time periods II/I & Number & $\%$ & $\% \quad(95 \% \mathrm{Cl})$ & Parts of the day & Time periods II/I \\
\hline 1 & All births $\geq 37$ weeks gestational age & 477813 & 100 & & 1369 & 100 & $2.87(2.72-3.03)$ & & $0.90(0.83-0.96)$ & 5045 & 100 & $10.6(10.3-10.9)$ & & $0.96(0.93-1.00)$ \\
\hline 2 & Excluded antepartum mortality & 649 & 0.1 & & 649 & 47.4 & & & & 649 & 12.9 & & & \\
\hline 3 & Other excluded patient categories ${ }^{1}$ ) & 102904 & 21.5 & & 267 & 19.5 & $2.60(2.30-2.92)$ & & $1.09(0.92-1.29)$ & 1378 & 27.3 & $13.4(12.7-14.1)$ & & $1.05(0.98-1.13)$ \\
\hline 4 & All STAS births ${ }^{2}$ ) & 374260 & 78.3 & & 453 & 33.1 & $1.21(1.10-1.32)$ & & $0.96(0.84-1.09)$ & 3018 & 59.8 & $8.1(7.8-8.4)$ & & $0.97(0.93-1.02)$ \\
\hline 5 & STAS births supervised by $1^{\text {st }}$ line & 170629 & 35.7 & 100 & 74 & 5.4 & $0.43(0.33-0.53)$ & & $1.07(0.77-1.47)$ & 545 & 10.8 & $3.2(2.9-3.5)$ & & $0.83(0.74-0.93)$ \\
\hline 6 & Daytime & 47220 & 9.9 & 27.7 & 21 & 1.5 & $0.44(0.25-0.63)$ & Reference & $1.65(0.84-3.25)$ & 157 & 3.1 & $3.3(2.8-3.8)$ & Reference & $0.98(0.79-1.21)$ \\
\hline 7 & Evening/night & 80823 & 16.9 & 47.4 & 32 & 2.3 & $0.40(0.26-0.53)$ & $0.89(0.51-1.54)$ & $0.76(0.48-1.20)$ & 262 & 5.2 & $3.2(2.9-3.6)$ & $0.98(0.80-1.19)$ & $0.74(0.63-0.86)$ \\
\hline 8 & Duty handover & 42586 & 8.9 & 25.0 & 21 & 1.5 & $0.49(0.28-0.70)$ & $1.11(0.61-2.03)$ & $1.41(0.74-2.70)$ & 126 & 2.5 & $3.0(2.4-3.5)$ & $0.89(0.70-1.12)$ & $0.88(0.69-1.11)$ \\
\hline 9 & Referrals $1^{\text {st }}$ line to $2^{\text {nd }}$ line during labour & 79376 & 16.6 & 100 & 162 & 11.8 & $2.04(1.73-2.35)$ & & $0.93(0.75-1.14)$ & 1052 & 20.9 & $13.3(12.5-14.1)$ & & $0.98(0.91-1.07)$ \\
\hline 10 & Daytime & 26447 & 5.5 & 33.3 & 52 & 3.8 & $1.97(1.43-2.50)$ & Reference & $0.94(0.65-1.36)$ & 323 & 6.4 & $12.2(10.9-13.5)$ & Reference & $1.08(0.93-1.26)$ \\
\hline 11 & Evening/night & 32901 & 6.9 & 41.4 & 75 & 5.5 & $2.28(1.76-2.79)$ & $1.16(0.81-1.65)$ & $1.06(0.77-1.45)$ & 470 & 9.3 & $14.3(13.0-15.6)$ & $1.17(1.02-1.35)$ & $0.95(0.84-1.07)$ \\
\hline 12 & Duty handover & 20028 & 4.2 & 25.2 & 35 & 2.6 & $1.75(1.17-2.33)$ & $0.89(0.58-1.36)$ & $0.72(0.47-1.10)$ & 259 & 5.1 & $12.9(11.4-14.5)$ & $1.06(0.90-1.25)$ & $0.94(0.79-1.10)$ \\
\hline 13 & Onset supervision of labour $1^{\text {st }}$ line & 250005 & 52.3 & 100 & 236 & 17.2 & $0.94(0.82-1.06)$ & & $0.94(0.79-1.12)$ & 1597 & 31.7 & $6.4(6.1-6.7)$ & & $0.91(0.85-0.97)$ \\
\hline 14 & Daytime & 73667 & 15.4 & 29.5 & 73 & 5.3 & $0.99(0.76-1.22)$ & Reference & $1.05(0.76-1.45)$ & 480 & 9.5 & $6.5(5.9-7.1)$ & Reference & $1.03(0.91-1.17)$ \\
\hline 15 & Evening/night & 113724 & 23.8 & 45.5 & 107 & 7.8 & $0.94(0.76-1.12)$ & $0.95(0.71-1.28)$ & $0.93(0.72-1.20)$ & 732 & 14.5 & $6.4(6.0-6.9)$ & $0.99(0.88-1.11)$ & $0.84(0.77-0.93)$ \\
\hline 16 & Duty handover & 62614 & 13.1 & 25.0 & 56 & 4.1 & $0.89(0.66-1.13)$ & $0.90(0.64-1.28)$ & $0.86(0.61-1.22)$ & 385 & 7.6 & $6.2(5.5-6.8)$ & $0.94(0.83-1.08)$ & $0.90(0.79-1.03)$ \\
\hline 17 & Onset supervision of labour $2^{\text {nd }} / 3^{\text {rd }}$ line & 124255 & 26.0 & 100 & 217 & 15.9 & $1.75(1.51-1.98)$ & & $0.91(0.75-1.10)$ & 1421 & 28.2 & $11.4(10.8-12.0)$ & & $1.01(0.93-1.09)$ \\
\hline 18 & Daytime & 41452 & 8.7 & 33.4 & 73 & 5.3 & $1.76(1.36-2.16)$ & Reference & $1.09(0.78-1.53)$ & 427 & 8.5 & $10.3(9.3-11.3)$ & Reference & $1.00(0.87-1.14)$ \\
\hline 19 & Evening/night & 51997 & 10.9 & 41.8 & 93 & 6.8 & $1.79(1.43-2.15)$ & $1.02(0.75-1.38)$ & $0.92(0.69-1.23)$ & 630 & 12.5 & $12.1(11.2-13.1)$ & $1.18(1.04-1.33)$ & $1.02(0.91-1.14)$ \\
\hline 20 & Duty handover & 30806 & 6.4 & 24.8 & 51 & 3.7 & $1.66(1.20-2.11)$ & $0.94(0.66-1.34)$ & $0.73(0.50-1.05)$ & 364 & 7.2 & $11.8(10.6-13.0)$ & $1.15(1.00-1.32)$ & $0.99(0.86-1.15)$ \\
\hline 21 & STAS births supervised by $2^{\text {nd }} / 3^{\text {rd }}$ line & 203631 & 42.6 & 100 & 379 & 27.7 & $1.86(1.67-2.05)$ & & $0.91(0.79-1.05)$ & 2473 & 49.0 & $12.1(11.7-12.6)$ & & $0.99(0.93-1.04)$ \\
\hline 22 & Daytime & 67899 & 14.2 & 33.3 & 125 & 9.1 & $1.84(1.52-2.16)$ & Reference & $1.01(0.79-1.29)$ & 750 & 14.9 & $11.1(10.3-11.8)$ & Reference & $1.03(0.93-1.14)$ \\
\hline 23 & Evening/night & 84898 & 17.8 & 41.7 & 168 & 12.3 & $1.98(1.68-2.28)$ & $1.07(0.85-1.35)$ & $0.97(0.78-1.20)$ & 1100 & 21.8 & $13.0(12.2-13.7)$ & $1.17(1.07-1.29)$ & $0.97(0.89-1.06)$ \\
\hline 24 & Duty handover & 50834 & 10.6 & 25.0 & 86 & 6.3 & $1.69(1.33-2.05)$ & $0.92(0.70-1.21)$ & $0.72(0.55-0.95)$ & 623 & 12.3 & $12.3(11.3-13.2)$ & $1.11(1.00-1.23)$ & $0.96(0.86-1.07)$ \\
\hline 25 & Non-teaching hospitals & 82935 & 17.4 & 100 & 154 & 11.2 & $1.86(1.56-2.15)$ & & $0.96(0.77-1.19)$ & 940 & 18.6 & $11.3(10.6-13.0)$ & & $1.03(0.94-1.23)$ \\
\hline 26 & Daytime & 28941 & 6.1 & 34.9 & 48 & 3.5 & $1.66(1.19-2.13)$ & Reference & $1.03(0.69-1.54)$ & 293 & 5.8 & $10.1(8.97-11.3)$ & Reference & $1.16(0.99-1.37)$ \\
\hline 27 & Evening/night & 33330 & 7.0 & 40.2 & 65 & 4.7 & $1.95(1.48-2.42)$ & $1.18(0.81-1.71)$ & $0.97(0.69-1.36)$ & 399 & 7.9 & $12.0(10.8-13.1)$ & $1.18(1.02-1.37)$ & $0.97(0.85-1.12)$ \\
\hline 28 & Duty handover & 20664 & 4.3 & 24.9 & 41 & 3.0 & $1.98(1.38-2.59)$ & $1.20(0.79-1.81)$ & $0.87(0.57-1.31)$ & 248 & 4.9 & $12.0(10.5-13.5)$ & $1.19(1.00-1.40)$ & $0.98(0.82-1.16)$ \\
\hline 29 & Teaching hospitals & 95996 & 20.1 & 100 & 170 & 12.4 & $1.77(1.50-2.04)$ & & $0.95(0.77-1.17)$ & 1167 & 23.1 & $12.2(11.5-12.9)$ & & $0.96(0.88-1.04)$ \\
\hline 30 & Daytime & 31184 & 6.5 & 32.5 & 58 & 4.2 & $1.86(1.38-2.34)$ & Reference & $1.04(0.72-1.50)$ & 351 & 7.0 & $11.3(10.1-12.4)$ & Reference & $0.96(0.83-1.12)$ \\
\hline 31 & Evening/night & 40827 & 8.5 & 42.5 & 77 & 5.6 & $1.89(1.47-2.31)$ & $1.01(0.72-1.42)$ & $1.08(0.78-1.50)$ & 525 & 10.4 & $12.9(11.8-14.0)$ & $1.14(1.00-1.31)$ & $0.96(0.85-1.08)$ \\
\hline 32 & Duty handover & 23985 & 5.0 & 25.0 & 35 & 2.6 & $1.46(0.98-1.94)$ & $0.78(0.52-1.19)$ & $0.67(0.44-1.04)$ & 291 & 5.8 & $12.1(10.8-13.5)$ & $1.08(0.92-1.26)$ & $0.93(0.80-1.10)$ \\
\hline 33 & Teaching hospitals with $\mathrm{NICU}^{3}$ ) & 24700 & 5.2 & 100 & 55 & 4.0 & $2.23(1.64-2.81)$ & & $0.72(0.51-1.02)$ & 366 & 7.3 & $14.8(13.3-16.3)$ & & $0.97(0.84-1.12)$ \\
\hline 34 & Daytime & 7774 & 1.6 & 31.5 & 19 & 1.4 & $2.44(1.35-3.54)$ & Reference & $0.87(0.47-1.61)$ & 106 & 2.1 & $13.6(11.1-16.2)$ & Reference & $0.91(0.70-1.17)$ \\
\hline 35 & Evening/night & 10741 & 2.2 & 43.5 & 26 & 1.9 & $2.42(1.49-3.35)$ & $0.99(0.55-1.79)$ & $0.75(0.45-1.24)$ & 176 & 3.5 & $16.4(14.0-18.8)$ & $1.20(0.95-1.53)$ & $1.00(0.82-1.23)$ \\
\hline 36 & Duty handover & 6185 & 1.3 & 25.0 & 10 & 0.7 & $1.62(0.62-2.62)$ & $0.66(0.31-1.42)$ & $0.51(0.24-1.09)$ & 84 & 1.7 & $13.6(10.7-16.5)$ & $1.00(0.75-1.32)$ & $0.99(0.74-1.34)$ \\
\hline & A & B & C & D & $\mathbf{E}$ & $\mathbf{F}$ & G & H & $\mathrm{K}$ & M & $\mathbf{N}$ & V & W & z \\
\hline & $\begin{array}{l}\left.{ }^{1}\right) \text { Other excluded patient categories: electi } \\
\text { multiple pregnancy and induction of labour } \\
{ }^{2} \text { ) STAS: Spontaneous onset of labour, after } \\
\text { period, Alive at the onset of labour, Single }\end{array}$ & $\begin{array}{l}\text { e caesarea } \\
\text { reaching th } \\
\text { hild }\end{array}$ & $\begin{array}{l}\text { In secti } \\
\text { he Term }\end{array}$ & & $\begin{array}{l}\left.{ }^{3}\right) \text { NICU: I } \\
\text { 4) Perinat } \\
\text { postpartt } \\
\text { 5) PMR: P }\end{array}$ & $\begin{array}{l}\text { Jeonat } \\
\text { al mor } \\
\text { Im mo } \\
\text { erinata }\end{array}$ & $\begin{array}{l}\text { Intensive Care Uni } \\
\text { lity: antepartum m } \\
\text { ality up to and incli } \\
\text { Mortality Rate }\end{array}$ & $\begin{array}{l}\text { ortality, intrapartu } \\
\text { ding } 7 \text { days after } \mathrm{b}\end{array}$ & mortality and & $\begin{array}{c}\text { Onset sec } \\
- \\
- \\
-\end{array}$ & $\begin{array}{r}\text { conds } \\
\text { Day } \\
\text { Eve } \\
\text { Dut }\end{array}$ & $\begin{array}{l}\text { e of labour / caesa } \\
\text { e: } 9.00 \text { to } 16.00 \\
\text { g/night: } 19.00 \text { to } 6 \\
\text { andover: } 6.00 \text { to } 9 .\end{array}$ & $\begin{array}{l}\text { arean section during } \\
.00 \\
.00 \text { and } 16.00 \text { to } 19 .\end{array}$ & \\
\hline
\end{tabular}


Table 3 Distribution of (post)-term births and associated adverse outcomes over the distinct (merged) context related patient (sub)groups in time period 2008-2010

\begin{tabular}{|c|c|c|c|c|c|c|c|c|c|c|c|c|c|c|}
\hline \multirow{2}{*}{\multicolumn{2}{|c|}{$\begin{array}{l}\text { Time period 2008-2010 (III) } \\
\text { Source: Netherlands Perinatal Registry }\end{array}$}} & \multicolumn{3}{|c|}{ Births (records) } & \multicolumn{3}{|c|}{ Incidence perinatal mortality ${ }^{4}$ ) } & \multicolumn{2}{|c|}{ Comparison \% PMR (RR 95\% CI) ${ }^{5}$ ) } & \multicolumn{3}{|c|}{ Incidence Apgar score < 7 (5 $\mathrm{min})$} & \multicolumn{2}{|c|}{ Comparison \%o Apgar < 7 (RR 95\% CI) } \\
\hline & & Number & $\%$ & $\%$ & Number & $\%$ & $\% \quad(95 \% \mathrm{Cl})$ & Parts of the day & Time periods III/I & Number & $\%$ & $\% \quad(95 \% \mathrm{Cl})$ & Parts of the day & Time periods III/I \\
\hline & 1 All births $\geq 37$ weeks gestational age & 486140 & 100 & & 1044 & 100 & $2.15(2.02-2.28)$ & & $0.67(0.62-0.73)$ & 5249 & 100 & $10.8(10.5-11.1)$ & & $0.98(0.95-1.02)$ \\
\hline 2 & Excluded antepartum mortality & 513 & 0.1 & & 513 & 49.1 & & & & 513 & 9.8 & & & \\
\hline & 3 Other excluded patient categories ${ }^{1}$ ) & 127656 & 26.3 & & 204 & 19.5 & $1.60(1.38-1.82)$ & & $0.67(0.56-0.80)$ & 1757 & 33.5 & $13.8(13.1-14.4)$ & & $1.08(1.01-1.57)$ \\
\hline 4 & All STAS births ${ }^{2}$ ) & 357971 & 73.6 & & 327 & 31.3 & $0.91(0.81-1.01)$ & & $0.72(0.63-0.83)$ & 2979 & 56.8 & $8.3(8.0-8.6)$ & & $1.00(0.95-1.05)$ \\
\hline & STAS births supervised by $1^{\text {st }}$ line & 155450 & 32.0 & 100 & 40 & 3.8 & $0.26(0.18-0.34)$ & & $0.63(0.43-0.93)$ & 511 & 9.7 & $3.3(3.0-3.6)$ & & $0.85(0.76-0.95)$ \\
\hline & $5 \quad$ Daytime & 42259 & 8.7 & 27.2 & 11 & 1.1 & $0.26(0.11-0.41)$ & Reference & $0.97(0.44-2.13)$ & 149 & 2.8 & $3.5(3.0-4.1)$ & Reference & $1.04(0.83-1.29)$ \\
\hline 7 & Evening/night & 74903 & 15.4 & 48.2 & 20 & 1.9 & $0.27(0.15-0.38)$ & $1.03(0.49-2.14)$ & $0.51(0.30-0.87)$ & 241 & 4.6 & $3.2(2.8-3.6)$ & $0.91(0.74-1.12)$ & $0.73(0.62-0.86)$ \\
\hline & Duty handover & 38288 & 7.9 & 24.6 & 9 & 0.9 & $0.24(0.08-0.39)$ & $0.90(0.37-2.18)$ & $0.67(0.30-1.52)$ & 121 & 2.3 & $3.2(2.6-3.7)$ & $0.90(0.71-1.14)$ & $0.94(0.74-1.19)$ \\
\hline & Referrals $1^{\text {st }}$ line to $2^{\text {nd }}$ line during labour & 80747 & 16.6 & 100 & 113 & 10.8 & $1.40(1.14-1.66)$ & & $0.64(0.51-0.80)$ & 984 & 18.7 & $12.2(11.4-12.9)$ & & $0.90(0.83-0.98)$ \\
\hline 0 & Daytime & 26096 & 5.4 & 32.3 & 38 & 3.6 & $1.46(0.99-1.92)$ & Reference & $0.70(0.47-1.04)$ & 289 & 5.5 & $11.1(9.8-12.3)$ & Reference & 1.15) \\
\hline 1 & Evening/night & 34531 & 7.1 & 42.8 & 49 & 4.7 & $1.42(1.02-1.82)$ & $0.97(0.64-1.49)$ & $0.87(0.61-1.24)$ & 459 & 8.7 & $13.3(12.1-14.5)$ & $1.20(1.04-1.39)$ & $0.88(0.78-1.00)$ \\
\hline 2 & Duty handover & 20120 & 4.1 & 24.9 & 26 & 2.5 & $1.29(0.80-1.79)$ & $0.89(0.54-1.46)$ & $0.53(0.33-0.85)$ & 236 & 4.5 & $11.7(10.2-13.2)$ & $1.06(0.89-1.26)$ & $0.85(0.72-1.00)$ \\
\hline 3 & Onset supervision of labour $1^{\text {st }}$ line & 236197 & 48.6 & 100 & 153 & 14.7 & $0.65(0.55-0.75)$ & & $0.65(0.53-0.79)$ & 1495 & 28.5 & $6.3(6.0-6.7)$ & & $0.90(0.84-0.96)$ \\
\hline 4 & $4 \quad$ Daytime & 68355 & 14.1 & 28.9 & 49 & 4.7 & $0.72(0.52-0.92)$ & Reference & $0.76(0.53-1.09)$ & 438 & 8.3 & $6.4(5.8-7.0)$ & Reference & ( $0.89-1.15)$ \\
\hline 5 & Evening/night & 109434 & 22.5 & 46.3 & 69 & 6.6 & $0.63(0.48-0.78)$ & $0.88(0.61-1.27)$ & $0.62(0.46-0.83)$ & 700 & 13.3 & $6.4(5.9-6.9)$ & $1.00(0.89-1.12)$ & $0.84(0.76-0.92)$ \\
\hline 6 & $5 \quad$ Duty handover & 58408 & 12.0 & 24.7 & 35 & 3.4 & $0.60(0.40-0.80)$ & $0.84(0.54-1.29)$ & $0.58(0.39-0.87)$ & 357 & 6.8 & $6.1(5.5-6.7)$ & $0.95(0.83-1.10)$ & $0.89(0.78-1.02)$ \\
\hline 7 & 7 Onset supervision of labour $2^{\text {nd }} / 3^{\text {rd }}$ line & 121774 & 25.0 & 100 & 174 & 16.7 & $1.43(1.22-1.64)$ & & $0.75(0.61-0.91)$ & 1484 & 28.3 & $12.2(11.6-12.8)$ & & $1.07(1.00-1.16)$ \\
\hline 8 & $3 \quad$ Daytime & 40262 & 8.3 & 33.1 & 62 & 5.9 & $1.54(1.16-1.92)$ & Reference & $0.95(0.67-1.36)$ & 474 & 9.0 & $11.8(10.7-12.8)$ & Reference & $1.14(1.00-1.30)$ \\
\hline 9 & Evening/night & 51181 & 10.5 & 42.0 & 75 & 7.2 & $1.47(1.13-1.80)$ & $0.95(0.68-1.33)$ & $0.75(0.55-1.02)$ & 667 & 12.7 & $13.0(12.1-14.0)$ & $1.11(0.98-1.24)$ & $1.09(0.98-1.22)$ \\
\hline 0 & Duty handover & 30331 & 6.2 & 24.9 & 37 & 3.5 & $1.22(0.83-1.61)$ & $0.79(0.53-1.19)$ & $0.54(0.36-0.80)$ & 343 & 6.5 & $11.3(10.1-12.5)$ & $0.96(0.84-1.10)$ & $0.95(0.82-1.10)$ \\
\hline 1 & 1 STAS births supervised by $2^{\text {nd }} / 3^{\text {rd }}$ line & 202521 & 41.7 & 100 & 287 & 27.5 & $1.42(1.25-1.58)$ & & $0.69(0.60-0.81)$ & 2468 & 47.0 & $12.2(11.7-12.7)$ & & $0.99(0.94-1.05)$ \\
\hline 2 & $2 \quad$ Daytime & 66358 & 13.6 & 32.8 & 100 & 9.6 & $1.51(1.21-1.80)$ & Reference & $0.83(0.64-1.07)$ & 763 & 14.5 & $11.5(10.7-12.3)$ & Reference & $1.07(0.97-$ \\
\hline & Evening/night & 85712 & 17.6 & 42.3 & 124 & 11.9 & $1.45(1.19-1.70)$ & $0.96(0$. & $0.71(0.56-0.89)$ & 1126 & 21.5 & $13.1(12.4-13.9)$ & 1.14 & 1.07) \\
\hline 4 & Duty handover & 50451 & 10.4 & 24.9 & 63 & 6.0 & $1.25(0.94-1.56)$ & $0.83(0.60-1.14)$ & $0.53(0.39-0.72)$ & 579 & 11.0 & $11.5(10.6-12.4)$ & $1.00(0.90-1.11)$ & $0.90(0.80-1.01)$ \\
\hline 5 & Non-teaching hospitals & 83152 & 17.1 & 100 & 116 & 11.1 & $1.40(1.14-1.65)$ & & $0.72(0.57-0.91)$ & 896 & 17.1 & $10.8(10.1-11.5)$ & & $0.98(0.89-1.07)$ \\
\hline & Daytime & 27998 & 5.8 & 33.7 & 37 & 3.5 & $1.32(0.90-1.75)$ & Reference & $0.82(0.54-1.26)$ & 275 & 5.2 & $9.8(8.7-11.0)$ & Reference & $1.13(0.95-1.33)$ \\
\hline & Evening/night & 34385 & 7.1 & 41.4 & 53 & 5.1 & $1.54(1.13-1.96)$ & 77-1.77) & 0.7 & 417 & 7.9 & $12.1(11.0-13.3)$ & 1.44) & 1.13) \\
\hline & Duty handover & 20769 & 4.3 & 25.0 & 26 & 2.5 & $1.25(0.77-1.73)$ & $0.95(0.57-1.56)$ & $0.55(0.34-0.88)$ & 204 & 3.9 & $9.8(8.5-11.2)$ & $1.00(0.84-1.20)$ & $0.80(0.67-0.96)$ \\
\hline 19 & Teaching hospitals & 95334 & 19.6 & 100 & 109 & 10.4 & $1.14(0.93-1.36)$ & & $0.61(0.48-0.78)$ & 1154 & 22.0 & $12.1(11.4-12.8)$ & & $0.95(0.88-1.03)$ \\
\hline & Daytime & 30737 & 6.3 & 32.2 & 43 & 4.1 & $1.40(0.98-1.82)$ & Reference & $0.78(0.52-1.16)$ & 356 & 6.8 & $11.6(10.4-12.8)$ & Reference & $0.99(0.86-1.15)$ \\
\hline & Evening/night & 40667 & 8.4 & 42.7 & 42 & 4.0 & $1.03(0.72-1.34)$ & $0.74(0.48-1.13)$ & $0.59(0.40-0.87)$ & 520 & 9.9 & $12.8(11.7-13.9)$ & $1.10(0.97-1.26)$ & $0.95(0.85-1.08)$ \\
\hline & Duty handover & 23930 & 4.9 & 25.1 & 24 & 2.3 & $1.00(0.60-1.40)$ & $0.72(0.44-1.18)$ & $0.46(0.28-0.75)$ & 278 & 5.3 & $11.6(10.3-13.0)$ & $1.00(0.86-1.17)$ & $0.89(0.76-1.05)$ \\
\hline & Teaching hospitals with $\mathrm{NICU}^{3}$ ) & 24035 & 4.9 & 100 & 62 & 5.9 & $2.58(1.94-3.22)$ & & $0.83(0.60-1.16)$ & 418 & 8.0 & $17.4(15.7-19.0)$ & & $1.14(0.99-1.31)$ \\
\hline & Daytime & 7623 & 1.6 & 31.7 & 20 & 1.9 & $2.62(1.48-3.77)$ & Reference & $0.93(0.51-1.71)$ & 132 & 2.5 & $17.3(14.4-20.2)$ & Reference & $1.15(0.90-1.47)$ \\
\hline & Evening/night & 10660 & 2.2 & 44.4 & 29 & 2.8 & $2.72(1.73-3.71)$ & $1.04(0.59-1.83)$ & $0.84(0.51-1.37)$ & 189 & 3.6 & $17.7(15.2-20.2)$ & $1.02(0.82-1.28)$ & $1.09(0.89-1.33)$ \\
\hline & Duty handover & 5752 & 1.2 & 23.9 & 13 & 1.2 & $2.26(1.03-3.49)$ & $0.86(0.43-1.73)$ & $0.71(0.35-1.43)$ & 97 & 1.8 & $16.9(13.5-20.2)$ & $0.97(0.75-1.26)$ & $1.23(0.92-1.65)$ \\
\hline & A & B & C & D & $\mathbf{E}$ & $\mathbf{F}$ & G & H & K & M & $\mathbf{N}$ & v & w & Z \\
\hline & $\begin{array}{l}\left.{ }^{1}\right) \text { Other excluded patient categories: electiv } \\
\text { multiple pregnancy and induction of labour } \\
{ }^{2} \text { ) STAS: Spontaneous onset of labour, after } \\
\text { period, Alive at the onset of labour, Single c }\end{array}$ & $\begin{array}{l}\text { e caesarea } \\
\text { reaching tt } \\
\text { hild }\end{array}$ & in sect & & $\begin{array}{l}\left.{ }^{3}\right) \text { NICU: } \\
{ }^{4} \text { ) Perina } \\
\text { postpart } \\
\text { 5) PMR: }\end{array}$ & $\begin{array}{l}\text { Neona } \\
\text { tal mol } \\
\text { um mo } \\
\text { Perinat }\end{array}$ & $\begin{array}{l}\text { Intensive Care Uni } \\
\text { lity: antepartum m } \\
\text { ality up to and incli } \\
\text { Mortality Rate }\end{array}$ & ding 7 days afte & $\begin{array}{l}\text { mortality and } \\
\text { th }\end{array}$ & $\begin{array}{c}\text { Onset se } \\
- \\
- \\
-\end{array}$ & $\begin{array}{r}\text { conds } \\
\text { Day } \\
\text { Eve } \\
\text { Dut }\end{array}$ & $\begin{array}{l}\text { ge of labour / caes } \\
\text { ne: } 9.00 \text { to } 16.00 \\
\text { g/night: } 19.00 \text { to } \\
\text { landover: } 6.00 \text { to } 9\end{array}$ & $\begin{array}{l}00 \\
00 \text { and } 16.00 \text { to } 19\end{array}$ & \\
\hline
\end{tabular}

eriod, Alive at the onset of labour, Single child 


\section{Number of patients}

Compared to the reference period (I), period II shows a reduction of the total number of births $\geq 37$ weeks (B1) and also the number of STAS births (B4). The number of STAS births supervised by the second or third line (B21) remains practically the same. At the same time, there has been an absolute and relative decline in the number of patients in the excluded categories (B3,C3). Time period III subsequently shows a slight increase in the total number of births (B1), but a further decrease in the number of STAS births (B4). The number of STAS births supervised by the second or third line also shows a slight decline, both absolutely and relatively (B21,C21). This is accompanied by a substantial absolute and relative increase in the number of patients in the excluded categories (B3,C3). All this results in a decrease in the proportion of births supervised by the first line $(36.4 \%, 35.7 \%, 32 \%)$ (C5).

\section{Population characteristics}

In the basic population, the differences in mother, labour and child characteristics between the three successive time periods are small (table not shown). Exceptions to this are the decrease in the proportion of pregnancies $\geq 42$ weeks in the periods I $(5.9 \%)$, II $(5.3 \%)$ and III $(3.2 \%)$, the decrease in the proportion of breech presentations $(4.4 \%, 4.1 \%, 3.6 \%)$ and the increase in the proportion of deliveries with epidural analgaesia $(5.3 \%, 7.8 \%, 13.1 \%)$.

\section{Distribution over the $24 \mathrm{~h}$ day}

In line with our basic assumption, in each of the three time periods the total group of patients who started labour under the supervision of the first line shows a distribution pattern that approximates the expected distribution of the deliveries over distinct parts of the day (D14,D15,D16). In almost all other (merged) context related patient groups there is a disproportional distribution of patients (records) between the 'daytime group' and the 'evening/night group'. In the group of STAS births supervised by the first line the proportion of the 'daytime group' in the periods I (28.3\%), II (27.7\%) and III $(27.2 \%)$ (D6) is increasingly smaller than expected $(29.2 \%)$. At the same time, in the group of referrals during labour this proportion is considerably larger than expected $(33.7 \%, 33.3 \%, 32.3 \%)$ (D10). The same applies to the patient group supervised from the onset of labour by the second or third line $(34.1 \%$, $33.4 \%, 33.1 \%$ ) (D18). The deviation from the expected distribution over the parts of the day is by far the largest in the group of non-teaching hospitals (D26,D30,D34).

\section{Incidence of perinatal mortality}

In the basic population the perinatal mortality rate decreases from 1616 cases in the reference period (I) to 1369 in period II and 1044 in period III (E1). The relative incidence of perinatal mortality also declines, in period II $(10 \%)$ as well as in period III $(33 \%)(\mathrm{G} 1, \mathrm{~K} 1)$.
The STAS population shows a similar pattern in the decrease of the relative incidence of perinatal mortality $(\mathrm{G} 4, \mathrm{~K} 4)$. Also, the relative incidence in the excluded patient categories shows a substantial decrease in time period III $(\mathrm{G} 3, \mathrm{~K} 3)$.

Compared to time period I, in the group of STAS births supervised by the second or third line, there has been a slight drop in relative incidence in period II (9\%) and a substantial decline $(31 \%)$ in period III $(\mathrm{G} 21, \mathrm{~K} 21)$. The decrease in period II mainly concerns the 'duty handover group' (28\%) (G24,K24), while the further decline in period III concerns the 'duty handover group' (47\%) as well as the 'evening/night group' (29\%) (G23,K23).

Between the distinct parts of the day the differences in the incidence of perinatal mortality are the highest in time period I. Thus, compared to the 'daytime group' the incidence in the 'evening/night group' is $12 \%$ higher (H23) and in the 'duty handover group' 28\% higher (H24). These differences are mainly caused by the group of non-teaching hospitals (H27,H28). In period III only the 'evening/night group' within the group of non-teaching hospitals shows a higher incidence than the reference group (17\%) (H27). It is noteworthy that within the group of teaching hospitals, none of the successive time periods shows a higher incidence of perinatal mortality in the 'evening/night group' (H31).

\section{Incidence Apgar score $<7$}

In the basic population the absolute incidence of the Apgar score $<7$ shows a decrease from 5558 cases in time period I to 5045 in period II, followed by an increase to 5249 in period III (M1). The relative incidence shows a similar pattern in successive periods (V1, $\mathrm{Z1)}$. The same applies to the relative incidence in the STAS population $(\mathrm{V} 4, \mathrm{Z} 4)$.

Similarly, in the group of STAS births supervised by the second or third line there are hardly any differences in relative incidence between the time periods I, II and III (V21,Z21). Compared to time period I there is, within this main group in period III, a slight decline in the incidence of the Apgar score $<7$ in the group of teaching hospitals (5\%) (V29,Z29) and an increase in the group of teaching hospitals with a NICU (14\%) (V33,Z33). The excluded patient categories also show a rise in incidence in period III $(8 \%)(\mathrm{V} 3, \mathrm{Z3})$.

Despite the almost unchanged incidence in the main groups there has been a substantial reduction in the differences in the incidence of the Apgar score $<7$ between the distinct parts of the day in the successive time periods (W). This levelling of incidence differences between the parts of the day is accompanied by an increase in incidence in most 'daytime groups' (V,W).

\section{DISCUSSION}

By developing a descriptive model, we have produced a tool that can be helpful in the systematic monitoring 
Development of adverse outcomes in STAS births supervised by $2 \mathrm{nd} / 3 \mathrm{rd}$ line

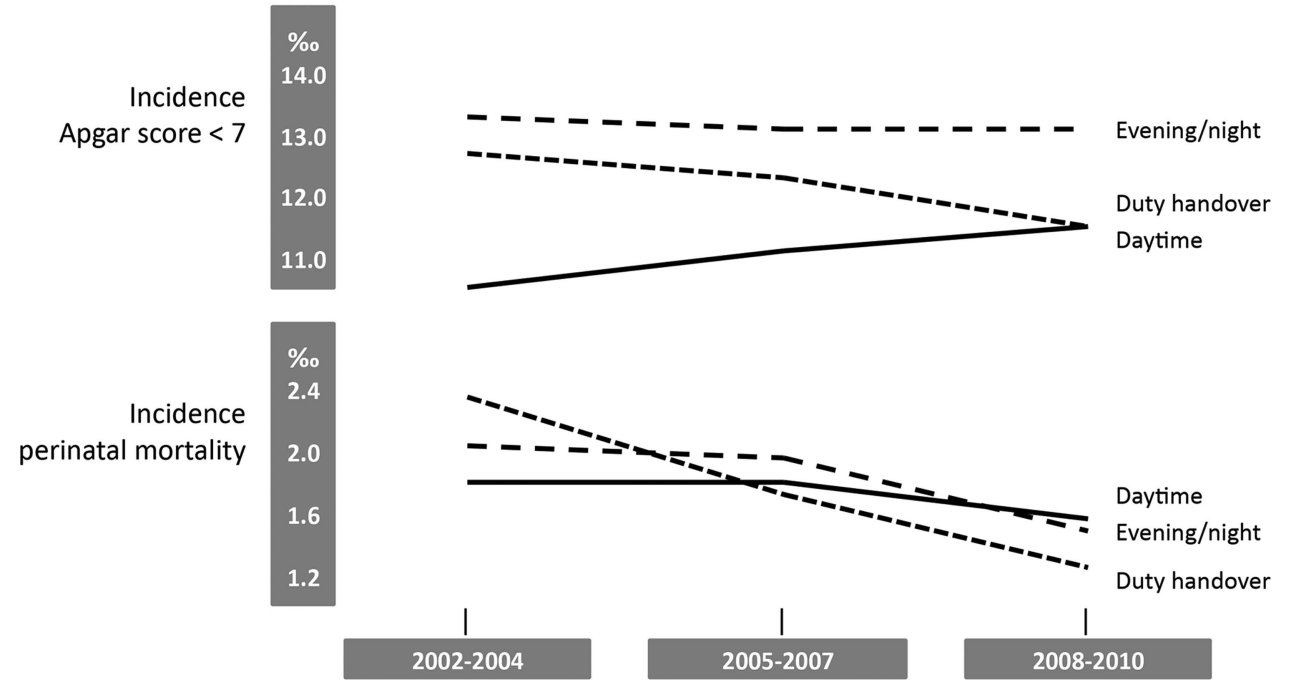

Figure 3 Development of adverse outcomes in Spontaneous onset of labour, after reaching the Term period, Alive at the onset of labour, Single child (STAS) births supervised by $2 \mathrm{nd} / 3 \mathrm{rd}$ line.

and evaluation of care in the obstetric care system. Even if the attention is focused on a part of the obstetric care system, the entire system remains in view. In this respect, our study design distinguishes itself from many other studies in this area. ${ }^{5-10}$ Yet there are more relevant differences. The design of the model is based on the most relevant organisational characteristics of the obstetric care system. In view of the system's dynamics we have opted for a combination of a transversal and a longitudinal study approach, while deliberately limiting the number of calendar years per distinct time period. A major limitation of this study is related to the common macro approach. The figures compared at the macro level consist of the sum of the figures that are collected at the meso level. This complicates the interpretation of the results. Small differences in the relative incidence of adverse outcomes at the macro level may hide much larger, in part mutually compensating, differences at the meso level. However, such a difference may equally well point to shortcomings in just a few units and/or wards.

\section{Diverging outcome variables}

Compared to the reference period, particularly in the most recent time period (2008-2010), the relative incidence of perinatal mortality in the term population is greatly diminished. In the STAS population this decline mainly concerns the 'evening/night groups' and the 'duty handover groups' (figure 3). As a result, there are hardly any demonstrable differences any more in the relative incidence of perinatal mortality between the parts of the day. It follows that such differences can no longer be used to question the safety of obstetric care outside office hours in the Netherlands. ${ }^{8} 16$

In contrast to the perinatal mortality rate, the incidence of the Apgar score $<7$ barely shows a decline in the successive time periods (figure 3). In most 'daytime groups' the levelling of the differences in incidence between the parts of the day is even accompanied by a slight rise in incidence. It is noteworthy that in the group of teaching hospitals with a NICU in the most recent time period, there has been an increase in the incidence of the Apgar score $<7$ during all parts of the day. It is not yet clear how this remarkable divergence of both outcome variables should be explained. The question of whether the quality of obstetric care in hospitals has improved, therefore, cannot be answered unequivocally.

\section{Complicating findings}

The presented results clearly support our basic assumption. In most (merged) context related patient groups, however, there is no proportional distribution of patients (records) over the distinct parts of the day. A plausible explanation is that the professional interventions that affect the course of childbirth are spread unevenly over a $24 \mathrm{~h}$ day. ${ }^{12}$ This applies first, to referrals from the first line to the second line during labour, and second, to the augmentation of labour under the supervision of the second or third line. This explains why the number of patients who reach the second stage of labour during daytime while being supervised by an obstetrician in the second/third line is proportionally greater than during the evening/night. Under these conditions, one cannot assume that the actual risk profiles of the 'daytime group' and the 'evening/night group' within the same (merged) context related patient group are equal to each other (figure 2).

To complicate matters, the absolute numbers of adverse outcomes on which the differences in relative incidence are based usually are very small. Thus a simple calculation shows that, in the most recent time period, the 'night/day difference' in the relative incidence of 
perinatal mortality in the total group of (about 40) nonteaching hospitals (RR 1.17) corresponds to three cases on an annual basis. Perhaps this is a good reason to consider the introduction of a new outcome variable that better matches the desired outcome of childbirth, for example: mother and child back home (in good health) within 1 week after birth.

Shifts between (merged) context related patient (sub) groups not only occur within a certain time period, but also in successive periods. Often these shifts are the result of new professional insights, standards and habits that lead to other referral patterns and/or interventions. Examples include the changed obstetric policy at breech presentations and at post-term pregnancies. With these types of changes over time the effect on the actual risk profile of the (merged) context related group can be assessed with reasonable accuracy. It is therefore easier to interpret a difference in the relative incidence of adverse outcomes by means of longitudinal comparisons than by means of transversal comparisons.

\section{CONCLUSION}

The complexity of the obstetric care system is not only the result of the multifactorial and dynamic character of the professional organisational contexts in which births take place. The size and the risk profile of the patient groups that are functionally related to these contexts are also in constant flux. This dynamic is to a large extent determined by professional intervention, at patient and also at policy level. All this makes it virtually impossible to demonstrate fixed patterns in the relationships between the separate contextual factors and the (adverse) outcomes of births. To generate useful knowledge it is necessary that changes in the obstetric care system are periodically and systematically evaluated. However, obstetrics is not practiced at the macro level. We should therefore consider complementing macro-level evaluations with parallel evaluations at the meso level. Here, too, our integral descriptive model could play a useful role.

Acknowledgements The authors thank the Netherlands Perinatal Registry for permission to use the registry data. They especially thank Chantal Hukkelhoven and Leanne Houweling for their help in extracting the data from the Netherlands Perinatal Registry. They also thank Paul van der Linden, Hans Merkus, Mathieu Weggeman and Ruud Jonkers for their critical remarks on the key concept of their study and on the manuscript.

Contributors LH and HS initiated and coordinated the study. LH coordinated data collection and performed quality control of data. HS designed the key concept. Both authors actively participated in interpreting the results and revising the paper.
Funding This research received no specific grant from any funding agency in the public, commercial or not-for-profit sectors.

Competing interests $\mathrm{LH}$ has been president of the board of the Netherlands Perinatal Registry until October 2013.

Provenance and peer review Not commissioned; externally peer reviewed.

Data sharing statement No additional data are available.

Open Access This is an Open Access article distributed in accordance with the Creative Commons Attribution Non Commercial (CC BY-NC 4.0) license, which permits others to distribute, remix, adapt, build upon this work noncommercially, and license their derivative works on different terms, provided the original work is properly cited and the use is non-commercial. See: http:// creativecommons.org/licenses/by-nc/4.0/

\section{REFERENCES}

1. Drife JO, Künzel W, Ulmsten U. The Peristat project. Eur J Obstet Gynecol Reprod Biol 2003;111:1-78.

2. Zeitlin J, Mohangoo A. The European Perinatal Health Report. Better statistics for better health for pregnant women and their babies. 2004. http://www.europeristat.com

3. Zeitlin J, Mohangoo A, Delnord M. The European Perintal Health Report. Health and Care of Pregnant Women and Babies in. 2010. http://www.europeristat.com

4. Kohn LT, Corrigan JM, Donaldson MS. To err is human. building a safer health system. Washington DC: National Academy Press, 2000.

5. Heller G, Misselwitz B, Schmidt S. Early neonatal mortality, asphyxia related deaths, and timing of low risk births in Hesse, Germany, 1990-8: observational study. BMJ 2000;21:274-5.

6. Luo ZC, Karlberg J. Timing of birth and infant and early neonatal mortality in Sweden 1973-95: Iongitudinal birth register study. BMJ 2001;323:1327-30.

7. Stephansson O, Dickman PW, Johansson AL, et al. Time of birth and risk of intrapartum and early neonatal death. Epidemiology 2003;14:218-22.

8. Visser GH, Steegers E. Beter baren. Nieuwe keuzen nodig in de zorg voor zwangeren (in Dutch). Medisch Contact 2008;3:96-9.

9. De Graaf JP, Ravelli AC, Visser GHA, et al. Increased adverse perinatal outcome of hospital delivery at night. BJOG 2010;117:1098-107.

10. Pasupathy D, Wood AM, Pell JP, et al. Time of birth and risk of neonatal death at term: retrospective cohort study. BMJ 2010;341: c3498.

11. World Health Organization. Conceptual Framework for the International Classification for Patient Safety. Final Technical Report -Version 1.1. January 2009.

12. Leeuw-Harmsen $L$ van der. Peripartale Organisatorische Context: een andere kijk op de verloskundige werkelijkheid (in Dutch). Grote lijnen, 10 jaar Perinatale Registratie Nederland, de grote lijnen. Edited by Stichting Perinatale Registratie 2011:66-9.

13. Anderka M, Declercq ER, Smith W. A time to be born. Am J Public Health 2000;90:124-6.

14. Mancuso PJ, Alexander JM, McIntyre DD, et al. Timing of birth after spontaneous onset of labor. Obstet Gynecol 2004;103:653-6.

15. Meray N, Reitsma JB, Ravelli AC, et al. Probabilistic record linkage is a valid and transparent tool to combine databases without a patient identification number. J Clin Epidemiol 2007;60:883-91.

16. Leeuw-Harmsen $\mathrm{L}$ van der, Schaaf JH: Perinatale sterfte vooral buiten kantooruren sterk afgenomen. Verloskundige zorg veiliger dan gedacht (in Dutch). Medisch Contact 2013;5:252-4.

17. Nicholl J. Case-mix adjustment in non-randomised observational evaluations: the constant risk fallacy. $J$ Epidemiol Community Health 2007;61:1010-30. 